\title{
Role of Cardiac Magnetic Resonance in Hypertrophic Cardiomyopathy
}

\section{Review Article}

Omar Deyaa Esmat Abdulkader ${ }^{1}$ (MSc); Sayed Ali Amin² (MD); Rehab Galal Abd El$\mathrm{Hmid}^{2}$ (MD); Salem Elsayed Deraz ${ }^{1,3}$ (MD);

1) Pediatric cardiology department, Magdy Yaacoub foundation (Aswan heart centre), Egypt

2) Pediatrics Department, Faculty of Medicine, Fayoum University, Egypt

3) Pediatrics Department, Faculty of Medicine, Menoufia University, Egypt

Corresponding Author

Omar Deyaa Esmat Abdulkader

Omardeyaa@yahoo.com

01002487046

\section{Abstract}

Hypertrophic cardiomyopathy is a complex inherited cardiovascular disease with wide phenotypic variation and a slow normal progression. Recent literature shows that cardiac magnetic resonance has increased its usefulness in the diagnosis, clinical preparation, and prognostication of this condition. Multimodality imaging of hypertrophic cardiomyopathy is being more widely used in clinical practice, and can help us to understand the subtle morphologic variations and their prognostic consequences.

Keywords:

Hypertrophic

Cardiomyopathy, Cardiac Magnetic

Resonance

\section{Introduction}

Cardiovascular magnetic resonance imaging (CMR) embraces several modalities that provide detailed information on cardiac morphology, ventricular function and myocardial tissue characteristics (1) (O'Hanlon $R$ et al.,2007).

CMR should be considered in patients with Hypertrophic cardiomyopathy (HCM) at their baseline assessment if local resources and expertise permit (2)(Borger, M et al.,2014)

1- Assessment of ventricular morphology and function

In patients with good echocardiographic images, CMR provides similar information on ventricular function and morphology, (3)(PuntmannVO et al.,2013) but it is helpful in establishing the diagnosis of HCM in patients with poor acoustic windows or when some Left ventricle (LV) regions are poorly visualized - such as the anterolateral wall, the LV 
apex and the right ventricle (4)(Rickers C et al.,2005), Cardiovascular magnetic resonance imaging is superior to transthoracic echocardiography (TTE) in the measurement of LV mass, but LV mass itself correlates weakly with maximal wall thickness and can be normal in patients with asymmetric HCM, especially when it involves less than two LV segments (3)(PuntmannVO et al.,2013). 
Cardiovascular magnetic resonance imaging is superior to standard two dimensional (2D) echocardiography in the detection of LV apical and anterolateral hypertrophy, aneurysms (5)(Maron MS et al.,2008) and thrombi (6)(Weinsaft JW et al.,2011), and is more sensitive in the detection of subtle markers of disease, such as myocardial crypts and papillary muscle abnormalities in patients with sarcomeric protein gene mutations (7)(Brouwer WP et al.,2012), (8)(Maron MS et al.,2012). Phase velocity flow mapping sequences can be used to determine the peak velocity of blood flow through the LV outflow tract in patients with left ventricular outflow tract obstruction (LVOTO), but proper alignment of the imaging plane, to obtain the highest flow velocities is time-consuming and prone to error. also the accurate quantification of turbulent flow is difficult and LV outflow gradients can only be measured at rest. For these reasons, Doppler echocardiography is the modality of choice for quantification of LVOTO. Similarly, while mitral inflow velocities and pulmonary vein flow derived from phase contrast CMR (PC-CMR) provide highly reproducible and accurate data in experienced hands, echocardiography is the preferred method for assessment of diastolic function in routine practice (9)(Nagueh SF et al.,2016). In selected cases where echocardiographic images are suboptimal, CMR is helpful in preoperative planning for surgical myectomy, particularly in patients with multi-level LV obstruction (LV outflow tract and mid-cavity) and in patients with right ventricular (RV) outflow tract abnormalities. CMR can also quantify the amount of tissue necrosis induced by septal alcohol ablation, as well as the location of scarring and the regression of LV mass following the procedure (10)(Yuan J et al.,2010).

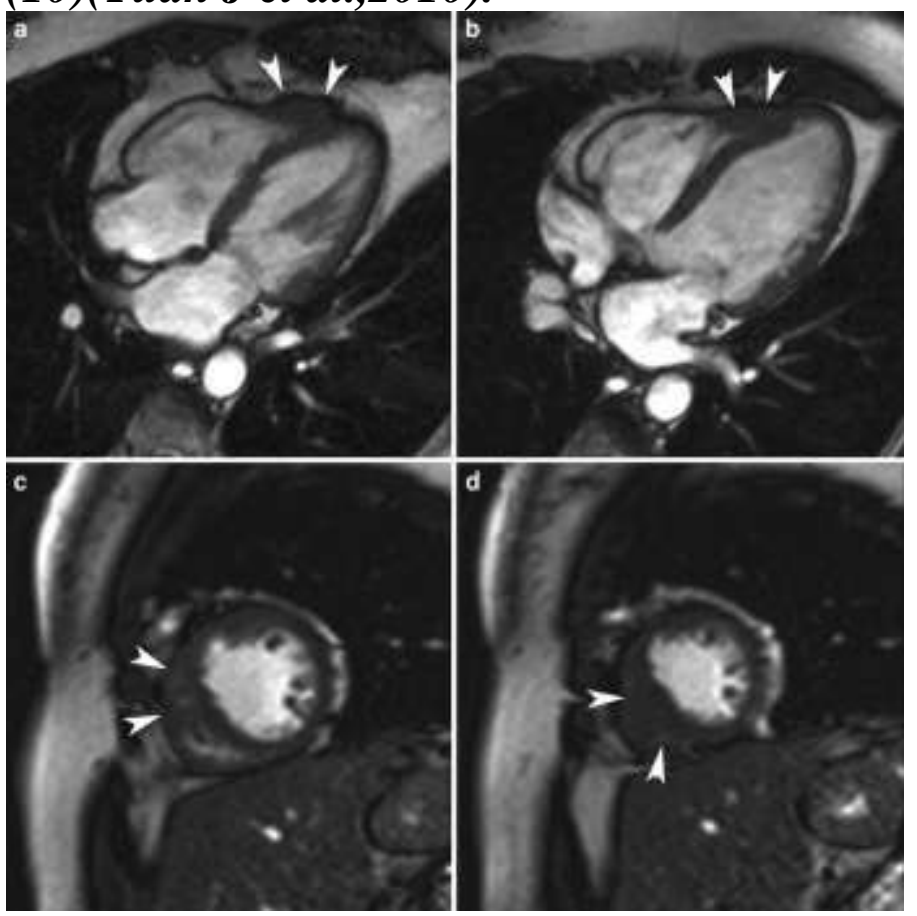

Figure 1: Focal form of hypertrophic cardiomyopathy in a patient with familial history of hypertrophic cardiomyopathy. Cine imaging in horizontal long-axis (a, b) and short- axis (c, d), showing enddiastolic (a, c) and end-systolic (b, d) time frames. The focal thickening of the apical $\mathrm{RV}$ free wall extending to the apical part of the ventricular septum can be well appreciated on MRI (arrowheads) (11)(Maron MS et al., 2009) 

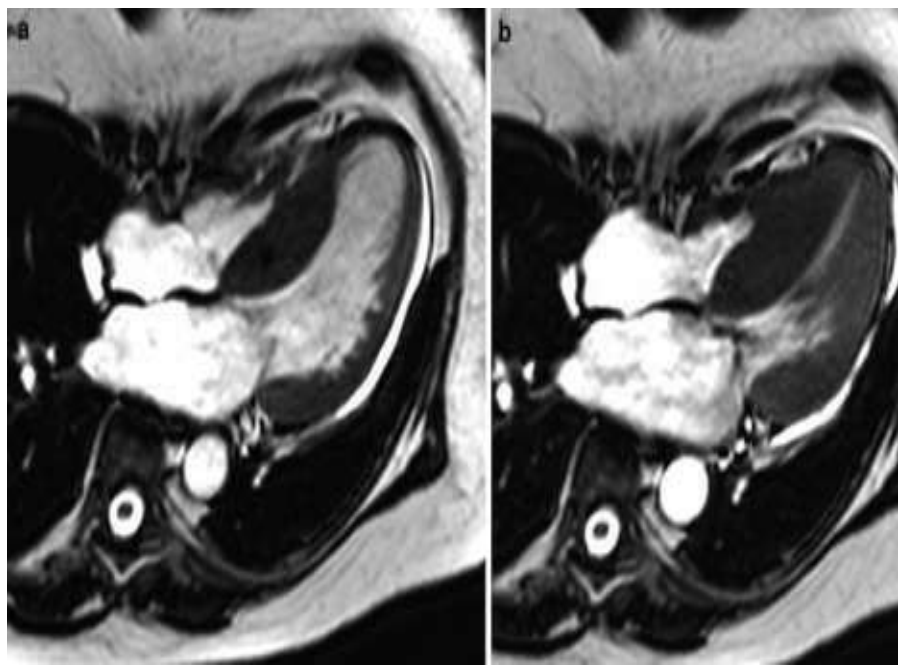

Figure 2: Asymmetrical septal HCM. Cine imaging at end diastole (a) and end systole

(b) in horizontal long-axis plane. The thickened appearance of the basal and mid-portionof the ventricular septum can be well appreciated on this horizontal long-axis view

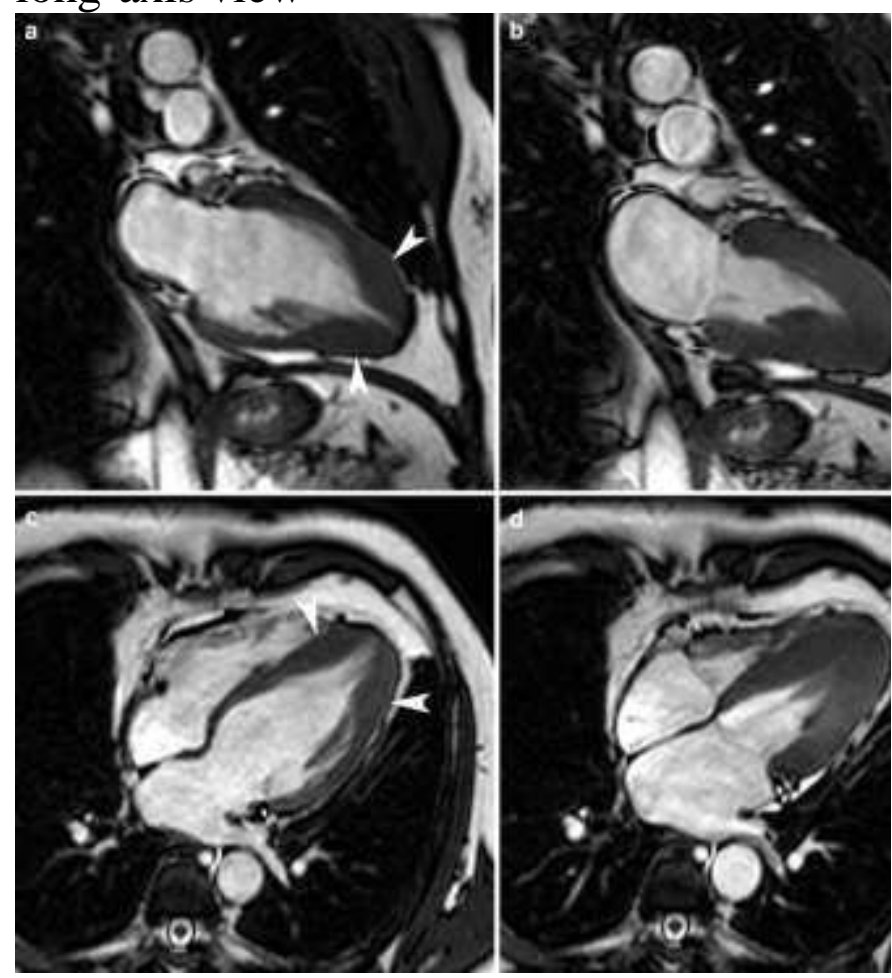

Figure 3: Apical form of HCM in a 54year-old man. Cine imaging in vertical $(\mathrm{a}, \mathrm{b})$ and horizontal long-axis plane (c, d), end-diastolic (a, c), and end-systolic (b, d) time frames. Circumferential thickening of the LV apex with maximal end diastolic wall thickness of $21 \mathrm{~mm}$

\section{(11)(Maron MS et al., 2009)}

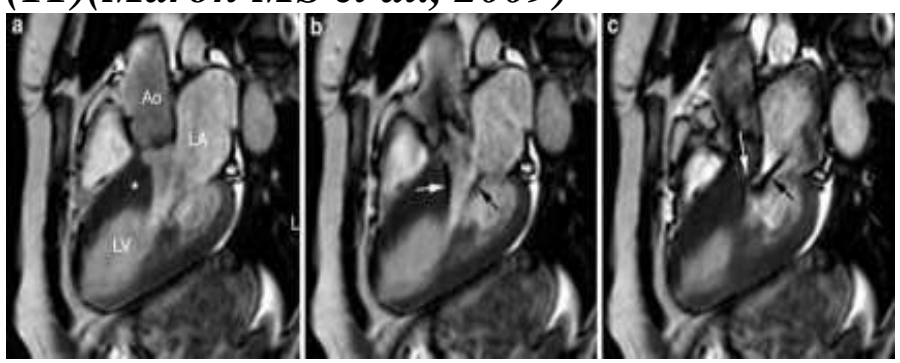

Figure 4: "Venturi' '-effect in hypertrophic (obstructive) cardiomyopathy. Cine imaging at end diastole (a), early systole (b), and mid systole (c). Important thickening of the basal ventricular septum $(*)$, causing LVOT narrowing. LV contraction leads to flow acceleration in the narrowed LVOT, visible as an area of signal void (arrow, b). The subsequent pressure drops cause SAM, leading to mitral regurgitation (black arrow,c) and left atrial enlargement

\section{2- Myocardial Fibrosis}

Myocardial fibrosis, a hallmark of hypertrophic cardiomyopathy, is thought to contribute to sudden cardiac death, ventricular tachy-arrhythmias, left ventricular dysfunction, and heart failure. Histologic evaluation universally reveals increased interstitial and focal myocardial fibrosis in overt disease. In most patients with hypertrophic cardiomyopathy, dense focal fibrosis can 
also be visualized noninvasively with the use of gadolinium-enhanced (CMR) (12)(Ho CY et al.,2010).

CMR can be used to detect expansion of the myocardial interstitium caused by fibrosis. Late gadolinium enhancement (LGE) is present in $65 \%$ of patients (range 33-84\%), typically in a patchy mid-wall pattern in areas of hypertrophy and at the anterior and posterior $\mathrm{RV}$ insertion points (13)(Rudolph $A$ et al.,2009) assessment of LGE before invasive treatment of LVOTO may be useful in selecting the most appropriate therapy by assessing the degree of septal fibrosis (2)(Borger, $M$ et al.,2014).

The trigger for increased myocardial fibrosis in hypertrophic cardiomyopathy remains unclear, but it has been attributed both to premature myocyte death caused by stresses imposed directly by sarcomere mutations and to later pathologic changes, including intracavitary obstruction, small-vessel disease, and ischemia, also studies showed that there is increased myocardial collagen synthesis in sarcomere-mutation carriers withoutovert disease, This profibrotic state preceded the development of left ventricular hypertrophy or fibrosis visible on MRI (12)(Ho CY et al.,2010).

The association between LGE and longterm outcomes has been examined in many studies, pooled data support a relationship between LGE and cardiovascular mortality, heart failure death and all-cause death, but show only a trend towards an increased risk of sudden cardiac death (SCD) (14)(Green JJ et al.,2012).
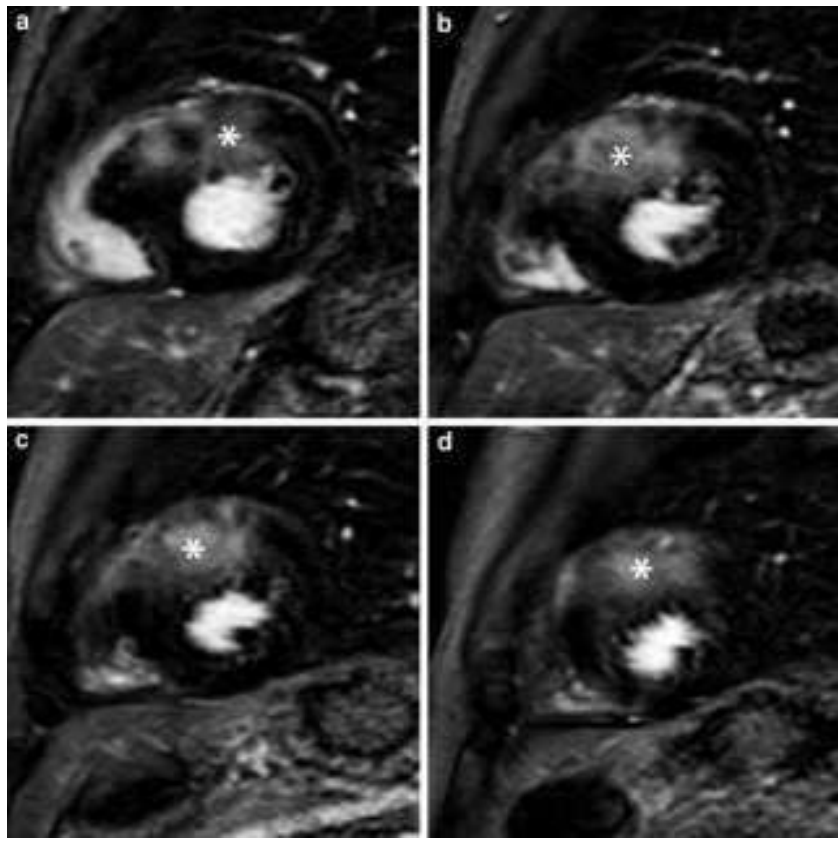

Figure 5: Typical enhancement pattern in HCM. Late Gd imaging in four shortaxis levels. Irregularly defined inhomogeneous 'cloudy' enhancement (*) in the thickenedmyocardium.

\section{3- $\quad$ Differential diagnosis}

Cardiac magnetic resonance imaging rarely distinguishes the causes of HCM by their magnetic properties alone, but the distribution and severity of interstitial expansion can, in context, suggest specific diagnoses. Anderson-Fabry disease is characterized by a reduction in noncontrast $\mathrm{T} 1$ signal and the presence of posterolateral LGE (15)(Sado DM et al.,2013). In cardiac amyloidosis, there is often global,sub-endocardial or segmental 
LGE and a highly specific pattern of myocardial and blood-pool gadolinium kinetics caused by similar myocardial and blood T1 signals (16)(Syed IS et al., 2010).

\section{References:}

1) O'Hanlon R, Assomull RG, Prasad SK. Use of cardiovascular magnetic resonance for diagnosis andmanagement in hypertrophic cardiomyopathy. Curr Cardiol Rep 2007;9:51-56.

2) Borger M, Charron P, Cecchi F, et al. 2014 ESC Guidelines on diagnosis and management of hypertrophic cardiomyopathy. Eur Heart J 2014;35:3322 .3332-

3) Puntmann VO, Gebker R, Duckett S, et al. Left ventricular chamber dimensions and wall thickness by cardiovascular magnetic resonance: comparison with transthoracic echocardiography. Eur Heart J Cardiovasc Imaging 2013;14:240-246.

\section{4) Rickers C, Wilke NM, Jerosch-Herold M, et}

al. Utility of cardiac magnetic resonance imaging in the diagnosis of hypertrophic cardiomyopathy. Circulation 2005;112:855861.

5) Maron MS, Finley JJ, Bos JM, et al. Prevalence, clinical significance, and natural history of left ventricular apical aneurysms in hypertrophic cardiomyopathy. Circulation 2008; 118:1541-1549.

6) Weinsaft JW, Kim HW, Crowley AL, et al. LV thrombus detection by routine echocardiography: insights into performance characteristics using delayed enhancement CMR. JACC Cardiovasc Imaging 2011;4:702712.

7) Brouwer WP, Germans T, Head MC, et al. Multiple myocardial crypts on modified long-axis view are a specific finding in prehypertrophic HCM mutation carriers. Eur Heart J Cardiovasc Imaging 2012;13:292-297.

8) Maron MS, Rowin EJ, Lin D, et al. Prevalence and clinical profile of myocardial crypts in hypertrophic cardiomyopathy. Circ Cardiovasc Imaging 2012;5:441-447.

9) Nagueh SF, Smiseth OA, Appleton CP, et al. Recommendations for the Evaluation of Left Ventricular Diastolic Function by Echocardiography: An Update from the American Society of Echocardiography and the European Association of Cardiovascular Imaging. J Am Soc Echocardiogr 2016;29:277314.

10) Yuan J, Qiao S, Zhang Y, et al. Followup by cardiac magnetic resonance imaging in patients with hypertrophic cardiomyopathy who underwent percutaneous ventricular septal ablation. AmJCardiol2010;106:1487-1491.

\section{1) Maron MS, Maron BJ, Harrigan C, et} al. Hypertrophic cardiomyopathy phenotype revisited after 50 years with cardiovascular magnetic resonance. J Am Coll Cardiol. 2009 14;54(3):220-8

\section{2) Ho CY, Lopez B, Otavio R. Coelho-}


Filho OR, et al. Myocardial Fibrosis as an

Early Manifestation of Hypertrophic

Cardiomyopathy. N Engl J Med

2010;363:552-63.

\section{3) Rudolph A, Abdel-Aty H, Bohl S, et al.}

Noninvasive detection of fibrosis applying contrast-enhanced cardiac magnetic resonance in different forms of left ventricular hypertrophy relation to remodeling. $\mathrm{J}$ Am Coll Cardiol 2009;53:284-291

\section{4) Green JJ, Berger JS, Kramer CM,}

Salerno M. Prognostic value of late gadolinium enhancement in clinical out comes for hypertrophic cardiomyopathy. JACC Cardiovasc Imaging 2012;5:370-377.

\section{5) Sado DM, White SK, Piechnik SK, et}

al. Identification and assessment of Anderson-

Fabry disease by cardiovascular magnetic resonance noncontrast myocardial $\mathrm{T} 1$ mapping. Circ Cardiovasc Imaging 2013;6:392-398.

\section{6) Syed IS, Glockner JF, Feng D, et al.}

Role of cardiac magnetic resonance imaging in the detection of cardiac amyloidosis. JACC Cardiovasc Imaging 2010;3:155-164. 23 
ISSN: 2536-9474 (Print)

ISSN: 2536-9482 (Online)

2021,8(4), 29-36
Original article / FYMJ

Fayoum University Medical Journal Amin et al., 